hep-th/0007241

RUNHETC-2000-29

\title{
Supergravity Solutions for Localised Brane Intersections
}

\author{
Arvind Rajaraman 网 \\ Department of Physics and Astronomy, Rutgers University, Piscataway, NJ 08855.
}

\begin{abstract}
We present a general method for constructing supergravity solutions for intersecting branes. The solutions are written in terms of a single function, which is the solution to a nonlinear differential equation. We illustrate this procedure in detail for the case of M2-branes ending on M5-branes. We also present supergravity solutions for strings ending on Dp-branes. Unlike previous results in the literature, these branes are completely localized.
\end{abstract}

*e-mail address: arvindra@muon.rutgers.edu 


\section{INTRODUCTION}

Supergravity solutions representing D-branes, NS 5-branes, and M-branes have been known for a long time now [1]. It is also known that strings can end on D-branes [2] and branes can end on other branes [3]. However, supergravity solutions representing such intersecting objects are, by and large, unknown.

Previous work in the literature has mostly focused on cases where one of the branes is delocalized in some direction [4,5]. There are only a few cases where all the branes have been localized [6 16], but these appear to be found on a case by case basis.

In this paper, we will provide a completely general method for finding supergravity solutions representing intersecting branes. This is based on the method introduced by 15 (See also [16]). Using this approach, which we describe in more detail below, we are able to write the solution in terms of the solution to a single nonlinear differential equation. Unfortunately, we have been unable to find explicit solutions of this differential equation. Nevertheless, we regard this as a solution in principle, and it is far simpler than attempting to solve the coupled Maxwell-Einstein equations.

The idea in this approach is to use the supersymmetry equations (the equations for conserved Killing spinors) as linear equations determining the field strengths in terms of the metric. This enables us to write all the fields in terms of the parameters of the metric.

It is then straightforward to substitute these expressions into the equations of motion. By the miracle of supersymmetry, we find that all the equations of motion simplify into a few simple equations. All the parameters can then be determined in terms of one function, which is in turn a solution to a nonlinear differential equation. Although we have been unable to find explicit solutions to this nonlinear equation, nevertheless, we emphasize that the solution is completely determined in principle by this differential equation.

We carry out these steps in great detail for the case of M2-branes ending on M5-branes. This method can be easily generalized to all other systems of intersecting branes in Mtheory and string theory, and as a further example, we present solutions for strings ending 
on Dp-branes. Finally, we list several open problems.

\section{M2-BRANES ENDING ON M5-BRANES}

\section{A. Setup}

The notation will be as follows. Indices with a tilde over them will denote curved (world) indices, and indices without a tilde will be tangent indices.

The M5-brane is oriented along the directions $x_{0}$ (time), $x_{1}, x_{2}, x_{3}, x_{4}, x_{5}$. The M2-brane is oriented along the directions $x_{0}, x_{1}, x_{6}$. Indices $(2,3,4,5)$ will be labeled by $a, b, \ldots$, and indices $(7,8,9,10)$ will be labeled $\alpha, \beta, \ldots$

The M5-brane and M2-brane act as sources for $a_{\alpha \beta \gamma}^{(3)}$ and $a_{016}^{(3)}$ respectively. Correspondingly, we expect the field strengths $f_{\alpha \beta \gamma \delta}, f_{\alpha \beta \gamma a}, f_{\alpha \beta \gamma 6}$, and $f_{01 a 6}, f_{016 \alpha}$ to be nonzero. Furthermore, the M2 brane acts as a source for the $U(1)$ field on the worldvolume of the M5-brane. If the worldvolume field strength is denoted by $h$, then $h_{01 a}$ and $h_{a b c}$ are nonzero. This field acts as a source for $a_{a b c}^{(3)}$ and $a_{01 a}^{(3)}$. Correspondingly, we also expect $f_{01 a \alpha}$, and $f_{2345}, f_{a b c 6}, f_{a b c \alpha}$ to be nonzero.

Also, since $\partial_{a} X^{6}$ is nonzero on the M5 brane worldvolume, we expect $g_{a 6}$ to be nonzero.

The nonzero components of the field strengths will be denoted

$$
F_{\alpha \beta \gamma \delta}, \quad F_{\alpha \beta \gamma a}, \quad F_{\alpha \beta \gamma 6} ; \quad G_{01 a 6}, \quad G_{01 a \alpha}, \quad G_{016 \alpha} ; \quad H_{2345}, \quad H_{a b c 6}, \quad H_{a b c \alpha}
$$

We have chosen a notation where different components of the field strength are denoted by $F, G$, or $H$ depending on the indices.

The symmetry further dictates that $\left(U=\sqrt{x_{\alpha}^{2}}\right)$

$$
\begin{aligned}
& F_{8910 a}=\frac{x_{7}}{U} F_{a} \quad F_{7910 a}=-\frac{x_{8}}{U} F_{a} \quad F_{7810 a}=\frac{x_{9}}{U} F_{a} \quad F_{789 a}=-\frac{x_{10}}{U} F_{a} \\
& F_{89106}=\frac{x_{7}}{U} F_{6} \quad F_{79106}=-\frac{x_{8}}{U} F_{6} \quad F_{78106}=\frac{x_{9}}{U} F_{6} \quad F_{7896}=-\frac{x_{10}}{U} F_{6} \\
& H_{a b c \alpha}=\frac{x_{\alpha}}{U} H_{a b c} \quad G_{016 \alpha}=\frac{x_{\alpha}}{U} G_{6} \quad G_{01 a \alpha}=\frac{x_{\alpha}}{U} G_{a}
\end{aligned}
$$




\section{B. The Equations for Supersymmetry}

We can now analyse the equation for conserved supercharges

$$
\delta \psi_{\tilde{\mu}}=\partial_{\tilde{\mu}} \epsilon-\frac{1}{4} \omega_{\tilde{\mu}}^{a b} \gamma_{a b}+\frac{i}{288}\left(\gamma_{\tilde{\mu}}^{\alpha \beta \gamma \delta}-8 \delta_{\tilde{\mu}}^{\alpha} \gamma^{\beta \gamma \delta}\right) f_{\alpha \beta \gamma \delta} \epsilon=0
$$

These equations are rather cumbersome. For example, for $\mu=1$, we have

$$
\begin{gathered}
\left(\frac{1}{2} \omega_{1}^{1 a} \gamma_{a}+\frac{1}{2} \omega_{1}^{16} \gamma_{6}+\frac{1}{2} \omega_{1}^{1 \alpha} \gamma_{\alpha}\right) \epsilon-\frac{i}{12} F_{78910} \gamma^{78910} \epsilon-\frac{i}{12} H_{2345} \gamma^{2345} \epsilon \\
-\frac{i}{12}\left(F_{789 a} \gamma^{789 a}+F_{7810 a} \gamma^{7810 a}+F_{7910 a} \gamma^{7910 a}+F_{8910 a} \gamma^{8910 a}\right) \epsilon \\
-\frac{i}{12}\left(F_{7896} \gamma^{7896}+F_{78106} \gamma^{78106}+F_{79106} \gamma^{79106}+F_{89106} \gamma^{89106}\right) \epsilon \\
-\frac{i}{12}\left(H_{234 \alpha} \gamma^{234 \alpha}+H_{235 \alpha} \gamma^{235 \alpha}+H_{245 \alpha} \gamma^{245 \alpha}+H_{345 \alpha} \gamma^{345 \alpha}\right) \epsilon \\
-\frac{i}{12}\left(H_{2346} \gamma^{2346}+H_{2356} \gamma^{2356}+H_{2456} \gamma^{2456}+H_{3456} \gamma^{3456}\right) \epsilon \\
+\frac{i}{6}\left(G_{01 a \alpha} \gamma^{01 a \alpha}+G_{01 a 6} \gamma^{01 a 6}+G_{016 \alpha} \gamma^{016 \alpha}\right) \epsilon=0
\end{gathered}
$$

All indices above are tangent space indices; we have defined $\omega_{a}^{b c}=e_{a}^{\tilde{\mu}} \omega_{\tilde{\mu}}^{b c}$.

To simplify the problem, we note that since we are looking for BPS solutions, we expect to be able to superpose them. In this case, we should be able to superpose M2 branes with arbitrary coordinates in the directions $x_{a}$. We can therefore separate the SUSY equations into terms which are even in $x_{a}$, and terms which are odd in $x_{a}$. These equations should be separately satisfied. The terms involving a $\partial_{a}$ clearly produces a term odd in $x_{a}$, while $\partial_{6}, \partial_{\alpha}$ produce terms even in $x_{a}$.

In addition, we will take the ansatz for the spinor to be

$$
\epsilon=\left(g_{11}\right)^{\frac{1}{4}} \epsilon_{0}
$$

where $\epsilon_{0}$ is a constant spinor. This ansatz is justified in [17].

The parts of the equations odd in $x_{4}$ are

$$
\begin{aligned}
\frac{1}{2} \omega_{1}^{14} \gamma_{4} \epsilon+\left(-\frac{i}{12} H_{235 \alpha} \gamma^{235 \alpha}-\frac{i}{12} H_{2356} \gamma^{2356}+\frac{i}{6} G_{014 \alpha} \gamma^{014 \alpha}+\frac{i}{6} G_{0146} \gamma^{0146}\right) \epsilon \\
-\frac{i}{12}\left(F_{7894} \gamma^{7894}+F_{78104} \gamma^{78104}+F_{79104} \gamma^{79104}+F_{89104} \gamma^{89104}\right) \epsilon=0
\end{aligned}
$$




$$
\begin{aligned}
& \frac{1}{2}\left(\omega_{5}^{54}-\omega_{1}^{14}\right) \gamma_{4} \epsilon+\left(\frac{i}{4} H_{235 \alpha} \gamma^{235 \alpha}+\frac{i}{4} H_{2356} \gamma^{2356}-\frac{i}{4} G_{014 \alpha} \gamma^{014 \alpha}-\frac{i}{4} G_{0146} \gamma^{0146}\right) \epsilon=0 \\
& \frac{1}{2}\left(\omega_{5}^{54} \gamma_{4}+\omega_{4}^{67} \gamma_{467} \epsilon\right)+\left(\frac{i}{4} H_{235 \alpha} \gamma^{235 \alpha}+\frac{i}{4} H_{2356} \gamma^{2356}\right) \epsilon \\
& \left(-\frac{i}{4} G_{014 \alpha} \gamma^{014 \alpha}-\frac{i}{4} G_{0146} \gamma^{0146}-\frac{i}{4} F_{89104} \gamma^{89104}\right) \epsilon=0 \\
& \frac{1}{2}\left(\omega_{6}^{64}-\omega_{1}^{14}\right) \gamma_{4} \epsilon-\frac{1}{2} \omega_{6}^{4 \alpha} \gamma_{64 \alpha} \epsilon+\left(\frac{i}{4} H_{2356} \gamma^{2356}-\frac{i}{4} G_{014 \alpha} \gamma^{014 \alpha}\right) \epsilon=0 \\
& -\frac{1}{2} \omega_{7}^{64} \gamma_{764}+\left(\frac{i}{4} G_{0147} \gamma^{0147}+\frac{i}{4} H_{2357} \gamma^{2357}-\frac{i}{4} F_{89104} \gamma^{89104}\right) \epsilon=0
\end{aligned}
$$

The parts of the equations even in all $x_{a}$ are

$$
\begin{aligned}
\frac{1}{2} \omega_{1}^{16} \gamma_{6} \epsilon+\frac{1}{2} \omega_{1}^{1 \alpha} \gamma_{\alpha} \epsilon+ & \left(\frac{i}{6} G_{016 \alpha} \gamma^{016 \alpha}-\frac{i}{12} H_{2345} \gamma^{2345}-\frac{i}{12} F_{78910} \gamma^{78910}\right) \epsilon \\
-\frac{i}{12}\left(F_{7896} \gamma^{7896}+F_{78106} \gamma^{78106}+F_{79106} \gamma^{79106}+F_{89106} \gamma^{89106}\right) \epsilon & =0 \\
\frac{1}{2}\left(\omega_{5}^{56}-\omega_{1}^{16}\right) \gamma_{6} \epsilon+\frac{1}{2}\left(\omega_{5}^{5 \alpha}-\omega_{1}^{1 \alpha}\right) \gamma_{\alpha} \epsilon+\left(\frac{i}{4} H_{2345} \gamma^{2345}-\frac{i}{6} G_{016 \alpha} \gamma^{016 \alpha}\right) \epsilon & =0 \\
\frac{1}{2}\left(\omega_{6}^{6 \alpha}-\omega_{1}^{1 \alpha}\right) \gamma_{\alpha} \epsilon+\frac{i}{4}\left(F_{7896} \gamma^{7896}+F_{78106} \gamma^{78106}+F_{79106} \gamma^{79106}+F_{89106} \gamma^{89106}\right) \epsilon & =0 \\
\frac{1}{2}\left(\omega_{1}^{17}-\omega_{8}^{87}\right) \gamma_{7} \epsilon+\frac{i}{4} G_{0167} \gamma^{0167} \epsilon-\frac{i}{4} F_{89106} \gamma^{89106} \epsilon & =0
\end{aligned}
$$

These equations should preserve one quarter of the supersymmetries. In other words, each equation should be proportional to a linear combination of $P_{1} \epsilon_{0}$ and $P_{2} \epsilon_{0}$, where $P_{1} \epsilon_{0}=$ $P_{2} \epsilon_{0}=0$ and $P_{1}, P_{2}$ are projection operators.

A glance at the equations (5) and (6) shows that the only possible choice of projection operators is (upto signs)

$$
\begin{array}{r}
P_{1} \epsilon_{0} \equiv\left(1+i \gamma^{678910}\right) \epsilon_{0}=0 \\
P_{2} \epsilon_{0} \equiv\left(1+i \gamma^{016}\right) \epsilon_{0}=0
\end{array}
$$

With these projection operators, we can reduce the matrix equations (5) and (6) to a set of algebraic equations. We will treat these algebraic equations as equations determinining the various field strengths as functions of the spin connections (and thereby implicitly as functions of the metric.)

The equations for the field strengths are then 


$$
\begin{array}{r}
G_{0164}=4 w_{1}^{14}+2 w_{5}^{54} \\
G_{0167}=2 w_{1}^{17}-2 w_{4}^{47} \\
G_{0147}=2 w_{4}^{67} \\
H_{2356}=4 w_{5}^{54}+2 w_{1}^{14} \\
H_{2357}=-2 w_{4}^{67} \\
H_{2345}=2 w_{4}^{46}-2 w_{1}^{16} \\
F_{48910}=-2 w_{4}^{67} \\
F_{78910}=-2 w_{4}^{46}-w_{1}^{16} \\
F_{68910}=4 w_{4}^{47}+2 w_{1}^{17}
\end{array}
$$

In addition, we get the constraints

$$
\begin{array}{r}
w_{1}^{16}+w_{4}^{46}+w_{7}^{76}=0 \\
w_{1}^{18}+w_{4}^{48}+w_{7}^{78}=0 \\
w_{1}^{14}+w_{5}^{54}+w_{7}^{74}=0 \\
w_{4}^{67}=w_{6}^{47}=-w_{7}^{64} \\
w_{6}^{67}+2 w_{4}^{47}=0 \\
w_{6}^{64}=2 w_{5}^{54}+2 w_{1}^{14}
\end{array}
$$

In all the equations above, all the indices are tangent space indices; we have defined $w_{c}^{a b}=e_{c}^{\tilde{\mu}} w_{\tilde{\mu}}^{a b}$.

We can solve all the constraints above by the metric ansatz

$$
\begin{array}{r}
e_{0 \tilde{0}}=e_{1 \tilde{1}}=\lambda^{-\frac{1}{3}} H^{-\frac{1}{6}} \\
e_{2 \tilde{2}}=e_{3 \tilde{3}}=e_{4 \tilde{4}}=e_{5 \tilde{5}}=\lambda^{\frac{1}{6}} H^{-\frac{1}{6}} \\
e_{6 \tilde{6}}=\lambda^{-\frac{1}{3}} H^{\frac{1}{3}} \\
e_{7 \tilde{7}}=e_{8 \tilde{8}}=e_{9 \tilde{9}}=e_{10 \tilde{0} 0}=\lambda^{\frac{1}{6}} H^{\frac{1}{3}} \\
e_{6 \tilde{a}}=\phi_{a} e_{6 \tilde{6}}
\end{array}
$$


with the constraint

$$
\partial_{6}\left(H \phi_{a}\right)=\partial_{a} H
$$

\section{The Equations of Motion}

We now look at the equations of motion. Away from the M5-brane, we can look at the vacuum equations of motion, which for the gauge fields is

$$
\partial_{\mu} f^{\mu \nu \rho \sigma}=\frac{1}{2 .(24)^{2}} \epsilon^{\nu \rho \sigma \tilde{a} \tilde{b} \tilde{c} \tilde{e} \tilde{e} \tilde{g} \tilde{h}} f_{\tilde{a} \tilde{b} \tilde{c} \tilde{d}} f_{\tilde{e} \tilde{f} \tilde{g} \tilde{h}}
$$

We can now substitute the field strengths found above into these equations. Remarkably, all the equations of motion collapse to the equations

$$
\partial_{a}\left(\phi_{a}\right)-\frac{1}{H} \partial_{6} \lambda-\frac{1}{2} \partial_{6} \phi_{a}^{2}=0
$$

and

$$
\partial_{\alpha}^{2}(H)+\partial_{6}^{2}(H \lambda)=0
$$

When we include sources, we need to modify the second equation. Defining

$$
\partial_{\alpha}^{2}(H)+\partial_{6}^{2}(H \lambda)=\partial_{6} Q
$$

we have

$$
\begin{array}{r}
\partial_{7} F_{a 8910}-\partial_{8} F_{a 7910}+\partial_{9} F_{a 7810}-\partial_{10} F_{a 789}-\partial_{a} F_{78910}=\partial_{a} Q \\
\partial_{\mu} G^{\mu 01 a}+\frac{1}{2 .(24)^{2}} \epsilon^{\tilde{0} \tilde{1} \tilde{a} \tilde{A} \tilde{B} \tilde{C} \tilde{D} \tilde{E} \tilde{F} \tilde{G} \tilde{H}} f_{\tilde{A} \tilde{B} \tilde{C} \tilde{D}} f_{\tilde{E} \tilde{F} \tilde{G} \tilde{H}}=\partial_{a} Q-i \phi \partial_{6} Q \\
\partial_{6}\left(\partial_{\mu} G^{\mu 016}+\frac{1}{2 .(24)^{2}} \epsilon^{\tilde{0} \tilde{1} \tilde{6} \tilde{A} \tilde{B} \tilde{C} \tilde{D} \tilde{E} \tilde{F} \tilde{G} \tilde{H}} f_{\tilde{A} \tilde{B} \tilde{C} \tilde{D}} f_{\tilde{E} \tilde{F} \tilde{G} \tilde{H}}\right)=-\partial_{a}^{2} Q
\end{array}
$$

$Q$ thus parametrizes the perturbation of the original M5-brane due to the addition of the M2-brane. 


\section{Linearized analysis}

At the linearized level, we can drop the terms involving $\phi$. If we then take

$$
\begin{array}{r}
\partial_{\mu} G^{\mu \tilde{0} \tilde{1} \tilde{a}}+\frac{1}{2 .(24)^{2}} \epsilon^{\tilde{\tilde{1}} \tilde{a} \tilde{A} \tilde{B} \tilde{C} \tilde{D} \tilde{E} \tilde{F} \tilde{G} \tilde{H}} f_{\tilde{A} \tilde{B} \tilde{C} \tilde{D}} f_{\tilde{E} \tilde{F} \tilde{G} \tilde{H}}=\delta\left(x_{\alpha}\right) \delta\left(x_{6}\right) h^{01 a} \\
\partial_{\mu} G^{\mu \tilde{0} \tilde{1} \tilde{\sigma}}+\frac{1}{2 .(24)^{2}} \epsilon^{\tilde{0} \tilde{1} \tilde{6} \tilde{A} \tilde{B} \tilde{C} \tilde{D} \tilde{E} \tilde{F} \tilde{G} \tilde{H}} f_{\tilde{A} \tilde{B} \tilde{C} \tilde{D}} f_{\tilde{E} \tilde{F} \tilde{G} \tilde{H}}= \begin{cases}\delta^{4}\left(x_{a}\right) \delta^{4}\left(x_{\alpha}\right), & x_{6} \geq 0 \\
0, & \text { else. }\end{cases}
\end{array}
$$

the above equations are satisfied provided

$$
\partial_{a} h^{01 a}=\delta\left(x_{a}\right)
$$

In other words, we have a gauge field strength on the M5-brane corresponding to the field of an electric charge on a line at $x_{a}=0$. Also, we have a membrane (the source for $\left.A_{016}\right)$ extending along the positive $x_{6}$ axis. This is exactly the configuration of a M2-brane ending on an M5-brane.

At the linearized level, the equations (24), (26) and (28) also simplify to

$$
\begin{aligned}
\partial_{6}\left(H \phi_{a}\right) & =\partial_{a} H \\
\partial_{a}\left(H \phi_{a}\right) & =\partial_{6} \lambda \\
\partial_{\alpha}^{2}\left(H \phi_{a}\right)+\partial_{a} \partial_{6}(H \lambda) & =\partial_{a} Q
\end{aligned}
$$

which can be simplified to the linear equation

$$
H \partial_{a}^{2} \lambda+\partial_{6}^{2} \lambda+\partial_{\alpha}^{2} \lambda=\frac{1}{\partial_{6}} \partial_{a}^{2} Q= \begin{cases}\delta^{4}\left(x_{a}\right) \delta^{4}\left(x_{\alpha}\right), & x_{6} \geq 0 \\ 0, & \text { else. }\end{cases}
$$

More generally, we have multiple M2 branes ending at different points on the M5-brane. The general solution, at the linear level, is then

$$
\lambda=\int d x^{\prime} K\left(x, x^{\prime}\right) \rho\left(x^{\prime}\right)
$$

where we have introduced the Green's function K, satisfying

$$
\left(H_{0} \partial_{a}^{2}+\partial_{6}^{2}+\partial_{\alpha}^{2}\right) K\left(x, x^{\prime}\right)=\delta\left(x-x^{\prime}\right)
$$


$H_{0}$ is the harmonic function describing the M5-branes without the M2-branes and $\rho\left(x^{\prime}\right)$ is the membrane source density.

The full nonlinear solution no longer has pointlike sources. The source $Q$ is now a nontrivial function of $x_{a}$ and $x_{6}$, and even the identification of $Q$ as a worldvolume field is a little problematic.

However, at long distances from the M2-branes, the linearized analysis still applies, since $\lambda$ is small. Also, very close to any M2-brane, where $\lambda$ diverges, the solution (39) should still apply, since the effects of the membrane dominate.

At the nonlinear level, we can then say that $\lambda$ is a solution to the equations (24), (26) and (28), subject to the condition that

$$
\lambda=\int d x^{\prime} K\left(x, x^{\prime}\right) \rho\left(x^{\prime}\right)
$$

both when $\lambda$ is small or when it is very large. This is expected to completely specify the solution, although we cannot explicitly solve the equations.

\section{E. Comments}

1. One might ask why one needs to look at the equations of motions at all. After all, once we impose the constraint (24), we have a solution that is supersymmetric. It is well known that a solution preserving a supersymmetry should also satisfy the equations of motion.

The point is that imposing the supersymmetry constraints does not completely specify the sources. The M2-branes along $x_{0}, x_{1}, x_{6}$, and the M5-branes along $x_{0}, x_{1}, x_{2}, x_{3}, x_{4}, x_{5}$, preserve the supersymmetries (7). But one can also add M5-branes oriented along $x_{0}, x_{1}, x_{7}, x_{8}, x_{9}, x_{10}$ without breaking any more supersymmetries. The equation (26) precisely sets the number of these M5-branes to zero, so that we have the sources we started with.

2. The second equation (28) determines the positions of the M5-brane and M2-brane sources. 
One expects on physical grounds that the M5-branes should not deform in the $x_{\alpha}$ directions. In this case, one should find that $Q=\delta^{4}\left(x_{\alpha}\right) Q_{1}\left(x_{a}, x_{6}\right)$.

It is however not clear how to show that the differential equations (24), (26), (28) are consistent with this expectation. One could, in principle, worry that when one tried to integrate in the equations from $x_{\alpha}=\infty$, one gets a singularity away from the origin. The solution would then be valid only outside this region (somewhat like the enhancon phenomenon [18]). It would be extremely surprising (at least to this author) if such a phenomenon should occur in this system, so we shall assume that in fact, $Q=\delta^{4}\left(x_{\alpha}\right) Q_{1}\left(x_{a}, x_{6}\right)$.

\section{F. Summary}

The solution for M2-branes ending on M5-branes is given by the metric (23)

$$
\begin{aligned}
d s^{2}= & \lambda^{-\frac{2}{3}} H^{-\frac{1}{3}}\left(-d x_{0}^{2}+d x_{1}^{2}\right)+\lambda^{\frac{1}{3}} H^{-\frac{1}{3}}\left(d x_{2}^{2}+d x_{3}^{2}+d x_{4}^{2}+d x_{5}^{2}\right) \\
& +\lambda^{\frac{1}{3}} H^{\frac{2}{3}}\left(d x_{7}^{2}+d x_{8}^{2}+d x_{9}^{2}+d x_{10}^{2}\right)+\lambda^{-\frac{2}{3}} H^{\frac{2}{3}}\left(d x_{6}+\phi_{a} d x_{a}\right)^{2}
\end{aligned}
$$

The field strengths are given in equations (8)-(16).

We also have the equations (24), (26), and (28) which determine the parameters

$$
\begin{array}{r}
\partial_{6}\left(H \phi_{a}\right)=\partial_{a} H \\
\partial_{a}\left(\phi_{a}\right)-\frac{1}{H} \partial_{6} \lambda-\frac{1}{2} \partial_{6} \phi_{a}^{2}=0 \\
\partial_{\alpha}^{2}(H)+\partial_{6}^{2}(H \lambda)=\delta^{4}\left(x_{\alpha}\right) \partial_{6} Q
\end{array}
$$

where $Q$ is a function of $x_{a}, x_{6}$.

Far away from the M2-branes, we have the boundary condition

$$
H \rightarrow H_{0} \quad \phi_{a} \rightarrow 0 \quad \lambda \rightarrow 1
$$

The first linear perturbation in $\lambda$ is

$$
\lambda=\int d x^{\prime} K\left(x, x^{\prime}\right) \rho\left(x^{\prime}\right)+1
$$


where $\rho\left(x^{\prime}\right)$ is the membrane source density, and $K\left(x, x^{\prime}\right)$ is the Green's function satisfying

$$
\left(H_{0} \partial_{a}^{2}+\partial_{6}^{2}+\partial_{\alpha}^{2}\right) K\left(x, x^{\prime}\right)=\delta\left(x-x^{\prime}\right)
$$

where $H_{0}$ is the harmonic function describing the M5-brane background without the M2branes. Furthermore, $\lambda$ is expected to behave as the above equation (47) in regions where $\lambda$ diverges.

The system of equations (24), (26), and (28) can further be simplified to the following equations

$$
\begin{array}{r}
H \phi_{a}=\partial_{a} \partial_{6} \tau \\
H=\partial_{6}^{2} \tau \\
\lambda=\partial_{a}^{2} \tau-H \phi_{a}^{2}
\end{array}
$$

where the function $\tau$ satisfies the nonlinear differential equation

$$
\partial_{\alpha}^{2} \tau+\partial_{6}^{2} \tau \partial_{a}^{2} \tau-\left(\partial_{a} \partial_{6} \tau\right)^{2}=\delta^{4}\left(x_{\alpha}\right) \frac{1}{\partial_{6}} Q
$$

\section{STRINGS ENDING ON DP-BRANES}

We can carry out a very similar analysis for strings ending on Dp-branes. We shall take the Dp-branes oriented along the directions $x_{0} \ldots x_{p}$, the string is oriented along $x_{0}, x_{9}$.

The Killing spinors preserved by this configuration are given by $\epsilon=\left(g_{00}^{1 / 4}\right) \epsilon_{0}$, where $\epsilon_{0}$ is a constant spinor satisfying

$$
\begin{gathered}
\gamma^{09} \epsilon_{0}=\eta_{1} \epsilon_{0} \\
\gamma^{01 . . p} \epsilon_{0}=\eta_{2} \epsilon_{0}
\end{gathered}
$$

where $\eta_{1}, \eta_{2}$ are constants.

The Killing equations can be written in the generic form

$$
\begin{array}{r}
w_{\mu}^{a b} \gamma_{a b} \epsilon+\left(a_{G} G_{\mu a b} \gamma^{a b}+b_{G} G_{a b c} \gamma_{\mu}^{a b c}\right) \epsilon+\left(a_{H} H_{\mu a_{1} . . a_{p-2}} \gamma^{a_{1} . . a_{p-2}}+b_{H} H_{a_{1} . . a_{p-1}} \gamma_{\mu}^{a_{1} . . a_{p-1}}\right) \epsilon \\
+\left(a_{F} F_{\mu a_{1} . . a_{p}} \gamma^{a_{1} . . a_{p}}+b_{F} F_{a_{1} . . a_{p}} \gamma_{\mu}^{a_{1} . . a_{p}}\right) \epsilon=0
\end{array}
$$


The metric components are found by noting that the diagonal components are the products of the corresponding component in the metric of the string and the p-brane metric (i.e. $g_{i i}=g_{i i}^{(\text {string })} g_{i i}^{(p-b r a n e)}$.) (We will work in the string frame)

$$
\begin{array}{r}
e_{0 \tilde{0}}=\lambda^{-\frac{1}{2}} H^{-\frac{1}{4}} \\
e_{1 \tilde{1}}=e_{2 \tilde{2}}=. .=e_{p \tilde{p}}=H^{-\frac{1}{4}} \\
e_{9 \tilde{9}}=\lambda^{-\frac{1}{2}} H^{\frac{1}{4}} \\
e_{(p+1)(p \tilde{+} 1)}=. .=e_{8 \tilde{8}}=H^{\frac{1}{4}}
\end{array}
$$

In addition, we have an off diagonal component.

$$
e_{9 \tilde{a}}=\phi_{a} e_{9 \tilde{9}}
$$

The dilaton is

$$
e^{\phi}=\lambda^{-1 / 2} H^{-\frac{p-3}{4}}
$$

The equations for the field strengths are then (all indices are tangent space indices)

$$
\begin{array}{r}
\left(a_{G} \eta_{1}\right) G_{019}=-w_{2}^{21}-w_{0}^{01} \\
\left(a_{G} \eta_{1}\right) G_{098}=w_{0}^{08}-w_{2}^{28} \\
\left(a_{G} \eta_{1}\right) G_{018}=w_{8}^{19} \\
\left(a_{H} \eta_{1} \eta_{2}\right) e^{\phi} H_{92 . . p}=-w_{2}^{21} \\
\left(a_{H} \eta_{1} \eta_{2}\right) e^{\phi} H_{82 . . p}=\frac{1}{2} w_{8}^{19} \\
\left(a_{H} \eta_{1} \eta_{2}\right) e^{\phi} H_{1 . . p}=\frac{1}{2}\left(w_{2}^{29}-w_{0}^{09}\right) \\
\left(a_{F} \eta_{2}\right) e^{\phi} F_{801 . . p}=w_{2}^{28} \\
\left(a_{F} \eta_{2}\right) e^{\phi} F_{8902 . . p}=-w_{8}^{19} \\
\left(a_{F} \eta_{2}\right) e^{\phi} F_{901 . . p}=\frac{1}{2}\left(w_{0}^{09}+w_{2}^{29}\right)
\end{array}
$$

The equations relating the various parameters are 


$$
\begin{array}{r}
\partial_{9}\left(H \phi_{a}\right)=\partial_{a} H \\
H^{-1} \partial_{9} \lambda+\frac{1}{2} \partial_{9}\left(\phi_{a}^{2}\right)=\partial_{a} \phi_{a} \\
\partial_{9}^{2}(H \lambda)+\partial_{\alpha}^{2}(H)=\delta\left(x_{\alpha}\right) \partial_{9} Q
\end{array}
$$

which can be simplified in terms of a single function $\tau$

$$
\begin{array}{r}
H=\partial_{9}^{2} \tau \\
H \phi_{a}=\partial_{a} \partial_{9} \tau \\
\lambda=\partial_{a}^{2} \tau-H \phi_{a}^{2}
\end{array}
$$

where $\tau$ satisfies

$$
\partial_{\alpha}^{2} \tau+\partial_{9}^{2} \tau \partial_{a}^{2} \tau-\left(\partial_{a} \partial_{6} \tau\right)^{2}=\delta\left(x_{\alpha}\right) \frac{1}{\partial_{9}} Q
$$

which is the same differential equation as in the previous section.

\section{OPEN QUESTIONS}

We have reduced the construction of intersecting brane systems to solving a single nonlinear partial differential equation. Much of the physics is contained in the solution to this equation, which, unfortunately, we have been unable to find exactly. It would be useful to be able to extract some information from the equation, for instance, to know whether it admits multiple solutions in some cases.

There are several potential applications of these solutions. By the AdS/CFT correspondence [19, some of these solutions can be mapped to configurations in strongly coupled gauge theories. For example, the solution for $k$ D-strings ending on $N$ D3-brane is dual to a configuration of $k$ monopoles in $S U(N) \mathcal{N}=4$ Yang-Mills theory. We can then extract quantities like the quark monopole potential etc. in this limit.

Similarly, these configurations, can be used to construct supergravity duals for any gauge theory that can be engineered by putting branes on orbifolds. These include pure $\mathcal{N}=2$ gauge theories and $\mathcal{N}=1$ gauge theories. One can also study solitons in these theories. 
One very interesting open question is to find the near extremal solutions. It is, of course, not possible to use the supersymmetry equations there, but the solutions found here should be a useful starting point to get the nonextremal solutions. Such solutions would give us information about the thermodynamics of theories with less supersymmetry.

\section{ACKNOWLEDGEMENTS}

This research was supported in part by DOE grant DE-FG02-96ER40559. 


\section{REFERENCES}

[1] G. T. Horowitz and A. Strominger, Nucl. Phys. B360, 197 (1991).

[2] J. Polchinski, Phys. Rev. Lett. 75, 4724 (1995) hep-th/9510017.

[3] A. Strominger, Phys. Lett. B383, 44 (1996) hep-th/9512059].

[4] G. Papadopoulos and P. K. Townsend, Phys. Lett. B380, 273 (1996) hep-th/9603087.

[5] D. Youm, Nucl. Phys. B556, 222 (1999).

[6] A. A. Tseytlin, Nucl. Phys. B475, 149 (1996) hep-th/9604035.

[7] A. A. Tseytlin, Mod. Phys. Lett. A11, 689 (1996) [hep-th/9601177].

[8] S. Surya and D. Marolf, Phys. Rev. D58, 124013 (1998) hep-th/9805121.

[9] M. Cvetic, H. Lu, C. N. Pope and J. F. Vazquez-Poritz, hep-th/0005246.

[10] K. Hosomichi, JHEP 0006, 004 (2000) hep-th/0002069.

[11] N. Itzhaki, A. A. Tseytlin and S. Yankielowicz, Phys. Lett. B432, 298 (1998) hepth/9803103].

[12] A. Hashimoto, JHEP 9901, 018 (1999) hep-th/9812159.

[13] A. Gomberoff and D. Marolf, JHEP 0002, 021 (2000) hep-th/9912184.

[14] D. Marolf and A. Peet, Phys. Rev. D60, 105007 (1999) hep-th/9903213.

[15] A. Fayyazuddin and D. J. Smith, JHEP 9904, 030 (1999) hep-th/9902210.

[16] A. Fayyazuddin and D. J. Smith, hep-th/0006060.

[17] R. Kallosh and J. Kumar, Phys. Rev. D56, 4934 (1997) hep-th/9704189.

[18] C. V. Johnson, A. W. Peet and J. Polchinski, Phys. Rev. D61, 086001 (2000) hepth/9911161. 
[19] J. Maldacena, Adv. Theor. Math. Phys. 2, 231 (1998) hhep-th/9711200. 